\title{
Is a common grouping mechanism involved in the phenomena of illusory continuity and stream segregation?
}

\author{
ALBERT S. BREGMAN, CONNIE COLANTONIO, and PIERRE A. AHAD \\ McGill University, Montreal, Quebec, Canada
}

\begin{abstract}
Two auditory phenomena-stream segregation and illusory continuity through a wide-band noise interruption-were studied to determine whether the same principles of perceptual organization applied to both. A cycle was formed of a repeating alternation of two short bursts of narrow-band noise $(\mathrm{NBN})$, one centered at a high frequency $(\mathrm{H})$ and the other at a low frequency $(\mathrm{L})$, with shorter bursts of wide-band noise (WBN) inserted between successive NBNs (H WBN L WBN H WBN...). In some conditions, listeners could hear a single NBN moving up and down behind the WBN bursts, although there was no NBN present with the WBN. Listeners rated the strength of this illusory continuity. Center frequency separation, rate of onsets, and bandwidth of the NBNs were varied. Increases in values of all three variables decreased illusory continuity. Other listeners rated the stream segregation of the $H$ and $L$ bands when successive NBNs were separated either by WBN bursts (as above) or by silences. The same three acoustic variables were manipulated. Increases in all three variables decreased the perception of a single stream. The similar disruptive effects on illusory continuity and on the onestream percept in the stream segregation task support the idea that both phenomena depend on a common preliminary process of linking together the parts of a sequence that have similar frequencies.
\end{abstract}

It has been proposed that while two auditory phenomena-illusory continuity and auditory stream segregationhave been studied separately, they are very closely related, since both involve the grouping of parts of a sound sequence: When all the sounds are grouped into a single stream (absence of segregation) in the stream segregation experiment, this is analogous to grouping the preinterruption and postinterruption sounds into a single unbroken sound in the illusory continuity experiment (Bregman, 1990, p. 361).

Stream segregation, in a typical experiment, occurs as follows: There is an alternation of pure tones of high and low frequencies. Listeners judge whether they hear the sequence as a single coherent sequence, or stream, or as breaking up into two streams of sound, one high in frequency and the other lower, each heard as a coherent sequence. Segregation into two streams increases as the frequency separation of the high and low tones increases and as the rate of onsets increases.

Illusory continuity has been known by many names: the auditory figure-ground effect (Thurlow, 1957), the acoustic tunnel (Vicario, 1960), auditory induction (Warren, Obusek, \& Ackroff, 1972), the continuity effect (in experiments on the pulsation threshold; e.g., Houtgast, 1972), perceptual restoration (Warren, 1984), and the continuity illusion (Bregman, 1990). An example can be produced by alternating a pure tone of a fixed frequency with a much

Correspondence should be addressed to A. S. Bregman, Department of Psychology, McGill University, 1205 Docteur Penfield Ave., Montreal, PQ H3A 1B1, Canada (e-mail: bregman@hebb.psych.mcgill.ca). louder noise burst that contains that frequency. Under certain conditions, the separate occurrences of the tone join up perceptually into a single long tone that appears to continue "behind" the interrupting noise (see Bregman, 1990, pp. 344-383). This means that the part of the tone that continues behind the noise has been restored by the perceptual process.

One theory of illusory continuity is that of Warren (1982, 1984), in which the perceptual restoration of the softer sound during the louder interrupting sound is seen as a compensation for masking. In Warren's theory, there is no mention of frequency relations that must hold between the sound that precedes the interruption and the one that follows it (in the example of the last paragraph, the tones were all of the same frequency), nor is any relation drawn between the continuity illusion and auditory stream segregation.

It has been argued by Bregman (1990) that, in illusory continuity (as compared with stream segregation), the perceptual grouping mechanism has determined not only that the separate bursts of tone should be grouped but that the grouped tone segments should be treated as a single tone, continuing unbroken behind the interrupting sound. This contrasts with the grouping that occurs in a stream segregation experiment. In the latter, although a sequence of high tones may be perceived as belonging together and may be segregated from the concurrent sequence of low tones, each individual tone is still heard as a separate sound.

The similarity between illusory continuity and stream segregation may be due to the fact that they address related problems faced by the auditory system in natural environments. One function of perceptual grouping is probably to 
isolate a sequence of related sounds (e.g., the individual pecks of a woodpecker) from other concurrent sounds. Another function may be to deal with intermittent masking, in which the listener obtains a series of glimpses of a single sound in a complex and fluctuating mixture. By a glimpse, we mean a brief period in which the interfering sounds are softer, and the auditory system can obtain a good estimate of the properties of the softer sound in isolation. Stream organization may help to integrate these glimpses into representations of enduring sounds. This integration is the natural-world counterpart of the sequential grouping that is encountered in the typical laboratory study of illusory continuity.

If the grouping of related sounds is used both to integrate a sequence of similar, but discrete, sounds coming from a single source (e.g., the individual impacts in the pecking of the woodpecker) and to integrate glimpses of a continuous but intermittently masked sound (e.g., a voice being intermittently masked by the pecking of the woodpecker), we would expect to find that stream organization worked in similar ways in the following two cases: (1) the typical stream segregation experiment, in which the material to be grouped is a sequence of discrete sounds interleaved with discrete sounds of another type, and (2) the typical illusory continuity experiment, in which the auditory system interprets the sequence of softer sounds as glimpses of a continuous sound that are obtained whenever the louder obliterating sound falls silent.

\section{STIMULI OF THE PRESENT EXPERIMENT}

The acoustic relations between the phenomena of stream segregation and illusory continuity can be appreciated by examining the stimulus pattern that was used in the present experiment. We used a high-frequency narrow-band noise (NBN) burst alternating repetitively with a low-frequency

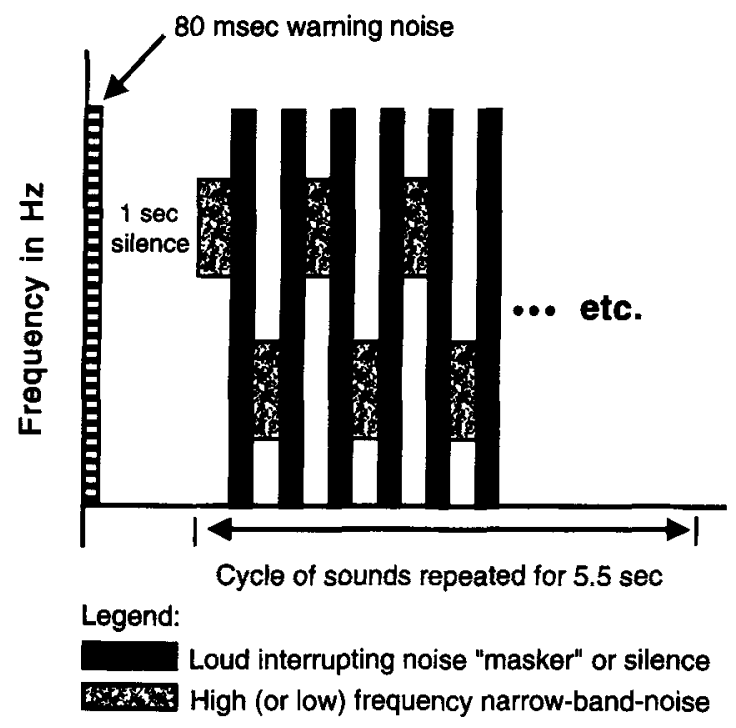

Figure 1. Stimulus pattern presented to listeners.
NBN burst, with bursts of wide-band noise (WBN) or silent gaps between them. Consider the case in which the WBN bursts are present (Figure 1). In preliminary testing, we determined that this stimulus can evoke stream segregation: Under some conditions, all the NBNs may be sequentially integrated into a single stream of alternating high and low noises; under others, the high-frequency NBNs may be segregated from the low-frequency ones so that we hear two streams, one composed of the high and the other composed of the low NBNs. (In both cases, the intermittent WBN can be heard as a separate stream.) The pattern can also give rise to illusory continuity: The NBNs are sometimes heard as parts of an unbroken NBN sound that is repeatedly rising and falling in frequency.

Both phenomena involve the segregation and integration that occur in perceptual organization. First, we consider segregation effects. In the stream segregation experiment, it occurs as the formation of distinct streams, whereas in the illusory continuity experiment, its presence is indicated by the experience that successive NBNs of alternating center frequencies are not connected to one another; in this case, segregation leads to a failure of continuity of the sequence as a whole.

Integration also occurs in both phenomena. In stream segregation, there is an integration of the component sounds that become parts of a single stream (e.g., the high tones). In illusory continuity, on the other hand, integration occurs when the bits of softer sound preceding and following the loud interruptions are put together with selected frequencies of the interrupting sound and are heard as parts of a single sound. Therefore, an individual stream in the stream segregation experiment is analogous to the perceived continuity of the interrupted sound in the illusory continuity experiment; in both cases, sounds are grouped together. The difference is that in illusory continuity, the grouped sounds are not heard as the discrete parts of a coherent sequence but as parts of a single unbroken sound. There has been only one study (Tougas \& Bregman, 1990) that has examined the relation between the two phenomena. It will be described later.

In discussing illusory continuity, let us call the softer sounds A and the loud interrupting ones B. Bregman (1990, pp. $349-370$ ) has outlined the properties that the sensory evidence must have before we will hear illusory continuity. These are summarized in four rules. (1) $A 1-A 2$ group ing rule: This is the requirement that $\mathrm{A} 1$, the softer sound that precedes the louder interruption (B), and $\mathrm{A} 2$, the softer sound that follows B, must be linked into a single stream, A. The qualities that promote successive A's being placed into the same stream include similarities in their spectral frequency region, fundamental frequency, location in space, and spectral distribution of components. (2) No discontinuity in $A$ rule: There should be no evidence that $A$ actually shuts off when B starts or turns on again when B finishes. (3) Sufficiency of evidence rule: During B, some subset of the neural activity in the auditory system should be indistinguishable from the activity that would have occurred if $A$ had actually continued. (4) $A$ is not $B$ rule: The 
transition from $A$ to $B$ and back again should not be interpretable as sound A transforming into a new form, B, and then back again. So, the grouping of $\mathrm{A} 1$ and $\mathrm{A} 2$ is one of four requirements in illusory continuity, whereas the sequential grouping of related elements is central in the phenomenon of stream segregation.

There are two possible relations between stream segregation and illusory continuity that would lead to the expectation that the same cues that affect sequential grouping in the stream segregation experiment should affect illusory continuity. The first possibility is that illusory continuity is a further refinement of stream formation. If this were true, the acoustic requirements for stream formation would automatically apply to illusory continuity. A second possibility is that the two phenomena merely represent different ways of dealing with the conclusion by the auditory system that certain bits of acoustic evidence go together. One way is to perceive them as parts of a series of sounds arising from a single acoustic source; the other is to experience them as parts of a single unbroken sound. Since both of these views of the relation between the two phenomena see them as depending on sequential grouping, the integration/segregation that is involved would be expected to be affected by the same acoustic variables in the two cases. The effects should be in the following direction: If an increase in any variable favors the segregation of a sequence into two streams (i.e., disrupts the integration of the whole sequence as a single stream), it should also decrease illusory continuity.

Such comparisons of phenomena are most effective when the same stimuli can be used for both. In experimenting with alternating high and low NBNs separated by WBN bursts, we discovered a pattern that could be segregated into high and low NBN streams and could also give rise to the experience of illusory continuity.

\section{THREE KEY VARIABLES}

We used the following three manipulations of the stimulus to affect the degree of integration of the sequence: (1) the rate of presentation of the sequence, (2) the separation of the center frequencies (CFs) of the high and low bands of noise, and (3) their bandwidths (BWs). Rate of presentation and frequency separation have long been known to affect stream segregation in research with sequences of tones. Both greater frequency separations and higher rates favor the segregation of a sequence into high and low streams (Bozzi \& Vicario, 1960; Bregman, 1990, chap. 2; van Noorden, 1975, 1977). Since NBNs have not been frequently used in stream segregation experiments, we manipulated $\mathrm{BW}$ to find out whether the effects of the other two variables required tone-like (very narrow-band) signals in order to have an effect.

NBNs were used only once before, to the best of our knowledge, to study stream segregation and illusory continuity. In the course of an experiment that was primarily about the subjective overlap of segregated streams, Dannenbring and Bregman (1976) alternated high and low
NBNs. These had two different CF separations: 3.2 and 19.0 semitones. Some listeners rated stream segregation, and others rated illusory continuity. Ratings on both phenomena were higher for the greater frequency separation. However, the experimenters concluded from certain anomalies in the results that illusory continuity had not really occurred but that the listeners had been rating the smooth transition of one NBN into the next, rather than the continuation of one behind the other (there was no WBN separating successive NBNs).

\section{EXPERIMENT}

In the present experiment, there were two tasks. The first asked a set of listeners to make ratings of illusory continuity. The second asked listeners to make judgments of stream integration/segregation. To make the two types of judgment comparable, we collected them on similar 7point rating scales: The value 1 referred to integration (single-stream perception) in the stream segregation task and to the perception of a single NBN moving up and down in frequency behind the WBN in the illusory continuity task, and the value 7 referred to two segregated streams in the stream segregation task and to a lack of illusory continuity in the illusory continuity task. We were afraid that if we gave the same subjects the task of judging both streaming and illusory continuity (on different trials), they might listen to the stimulus on any given trial in both ways. Since we wanted the judgments to be as independent as possible, the two tasks were run on separate groups of listeners who received instructions about, and clear examples of, the phenomenon they were to judge and heard no mention of the other phenomenon.

In addition to the illusory continuity task, there were two versions of the stream segregation task. The first was the typical task in which high- and low-frequency NBNs were alternated against a background of silence. Although this task was typical, it did not contain the WBNs that served as the loud interruptions that were required for the illusory continuity task. However, it was necessary to assess whether the presence of the WBNs altered stream organization in some essential way such that the comparison between the stream segregation and continuity-rating task was not valid. Therefore, we also included a task in which a separate group of listeners was asked to rate the integration/ segregation of the NBNs when these were separated by WBNs, as they were in the continuity-rating task. The resulting tasks are referred to as the illusory continuity task, the streaming with WBNs present task, and the streaming without WBNs task, respectively.

A second reason for including the WBNs in one version of the stream segregation task was that it has been proposed that "adding a loud masking noise between two sounds, A1 and A2, should never affect the way they enter into streams" (Bregman, 1990, p. 362). This proposal was supported in a study by Tougas and Bregman (1990), whose stimuli were an $\mathrm{X}$ pattern, consisting of a descending tonal glide simultaneous with an ascending tonal glide, each 
$1.6 \mathrm{sec}$ in duration, the two crossing in the center at time $t(0.8 \mathrm{sec})$. At this crossing point, the glides were (1) continuous right through it, (2) deleted from a $200-\mathrm{msec}$ interval centered at $t$ and replaced by silence, or (3) deleted from a $200-\mathrm{msec}$ interval centered at $t$ and replaced by a WBN. Listeners rated whether the ascending and descending glides crossed one another or seemed to bounce apart at the crossover point. The similarity of the timbres of the ascending and descending glides was manipulated. The pattern of results was similar regardless of the presence of the glides, silence, or noise at the crossover point. Listeners made no explicit judgments concerning the continuity illusion (whether they seemed to hear the glides behind the noise).

\section{METHOD}

\section{Subjects}

Originally, 53 listeners were recruited from a university population, but data from 5 were discarded and replaced in the course of running the experiment due to mistakes in the experimental procedure, leaving data from 48 ( 22 males and 26 females), or 16 listeners per task. These ranged in age from 18 to 45 years $(M=24, S D=$ 5). The subjects were paid to participate.

\section{Tasks}

Stimuli were NBN bursts and WBN bursts. Two NBN bursts repeatedly alternated on each trial, one having a higher (H) CF and the other a lower $(\mathrm{L})$ one. The stimulus sequence is shown in Figure 1. Three tasks were used, as described below, each employing a separate set of listeners.

Task 1: Ratings of illusory continuity. In Task 1, high and low NBNs alternated rapidly, with brief WBN bursts between them (e.g., H WBN L WBN H WBN L WBN ...). The listeners were asked to judge the degree to which the NBN was continuously present behind the WBN, moving up and down in frequency. Each trial began with an 80-msec burst of white noise as a warning signal, then $1 \mathrm{sec}$ of silence, then 5.5-sec worth of cycles of the stimulus pattern. After each trial, the listeners answered the question, "How clearly can you hear the high and low sounds connecting smoothly right through the noise?" Their responses were made on a 7-point scale, ranging from 1 (very clearly) to 7 (not very clearlv). Note that the low number represents continuity (integration), and the high number represents discontinuity (segregation).

Task 2: Streaming with WBNs present. In Task 2, the same stimuli were used as in Task 1, but the listeners were told that they would hear a simple melody made up of alternating high- and lowfrequency sounds. Bursts of loud noise, like "static," would also be present, but the listeners were to ignore them. Sometimes they would be able to follow a single up-and-down melody formed by the high and low sounds; at other times, they would hear the high and low sounds as forming separate streams. After each trial, the listeners answered the question, "How difficult is it to hold onto this simple melody?" Their responses were made on a 7-point scale, ranging from 1 (very easy) to 7 (very hard). Note that the low number represents integration of $\mathrm{H}$ and $\mathrm{L}$ sounds, and the high number represents segregation.

The reason for the use of this question as an index of segregation is that when a sequence segregates into two streams, the perception of patterns that must be followed across streams is disrupted (Bregman \& Campbell, 1971). Van Noorden (1975) showed that when a repeated galloping pattern of high and low tones (high-low-high, high-low-high, etc.) segregates into two streams, the high-lowhigh pattern disappears and is replaced by separate high and low streams, each containing repetitions of only a single pitch. Van Noorden also showed that only if the listener tries to hold on to the upand-down pattern as a single stream will the typical effects of frequency separation and rate of presentation be observed.

Task 3: Streaming without WBNs. In Task 3, the same task and stimuli were used as in Task 2, except that the WBN bursts were replaced by silences. The instructions were also the same, except that there was no mention of a loud static-like sound. This was the same as a typical test of stream segregation, except that the stimuli were $\mathrm{H}$ and $\mathrm{L}$ noise bursts rather than tones.

In each task, there were 18 practice trials (not scored), containing a different random selection of conditions for each listener. Then, there were two blocks of test trials, each consisting of one replication of each condition.

\section{Stimuli}

Frequency separation. Frequency separation is one of the strongest variables to affect stream segregation. Therefore, we used five levels of separation between the CFs of the high and low bands on a given trial: $3,8,13,18$, and 23 semitones. The low $C F$ was fixed at $1000 \mathrm{~Hz}$. The highest $\mathrm{CF}$ was $3780 \mathrm{~Hz}$.

Durations. It is well known that when high and low tones are presented in a cycle, stream segregation is sensitive to the rate of presentation. Therefore, we employed three different rates to study their effect both on stream segregation of NBN bursts and on their illusory continuity behind the WBN interruptions. The rate was changed by proportionally shortening the durations of all elements in the cycle, both NBNs and WBNs. The duration of each NBN was one of the following: $133 \mathrm{msec}$ (slow), $99 \mathrm{msec}$ (medium), or $73 \mathrm{msec}$ (fast). The duration of the WBNs in each condition was always half that of the NBNs. Therefore, all durations in the sequence were reduced proportionately to obtain the faster rates. Accordingly, the onset-to-onset times of successive NBNs were 200,148, and $110 \mathrm{sec}$ for the three conditions, respectively.

On each trial, the total exposure time to the cyclic pattern was fixed at approximately $5.5 \mathrm{sec}$. This meant that the number of presented cycles was greater for the shorter duration patterns; there were 14,19 , and 25 repetitions of the high-low pattern (i.e., H WBN L WBN) for the slow, medium, and fast conditions, respectively. Since the effects of greater numbers of exposures and faster cycles were correlated, this might be seen to raise a problem of interpretation. However, the goal of the experiment was to see whether factors known to promote stream segregation would reduce illusory continuity. Since both these correlated factors are known to increase stream segregation, the predictions for both stream segregation and illusory continuity were unambiguous - an increase of segregation in both cases.

NBN stimuli, bandwidth, and pitch strength. The NBN and WBN bursts had noise spectra that were "rectangular"-- the shape visible when plotting energy against frequency. Such bands have a spectrum in which all frequencies between the upper and lower band edge are of equal intensity (on average), and there is no energy outside these limits. We chose such spectra as a result of our preliminary research, which had used NBNs created by feeding white noise through digital resonators. Alternating sounds with the resulting spectra had not always led to strong segregation when their CFs were moved apart. We suspected that the problem was the imprecise nature of the frequency separation, due to the spectral skirts of the alternating bands of noise, which allowed some spectral overlap between the alternating bands. Hence, we chose rectangular noise bands, in which it is easy to specify the frequency separation (and also the percent spectral overlap, if any).

A second reason for using rectangular bands was that, in the illusory continuity task, we wanted to try to use NBNs that had as high an intensity as possible, because we believed that certain anomalies in preliminary research were attributable to excessive masking of the NBNs by the more intense WBN that we had used as an interrupting sound. Our preliminary research had used very loud WBN 
bursts because earlier research had done so (reviewed by Warren, $1982,1984)$. In relation to the required intensity of the WBN, Bregman $(1990$, p. 354) summarized the existing evidence as indicating that the mechanism that yields illusory continuity works by finding enough energy in the interrupting sound (B) at the frequencies of the softer sound $(A)$ without regard to the overall intensity of the interrupting sound. This is a conclusion that is consistent with statements by Warren et al. (1972), Warren (1984), and Houtgast (1972). It follows that the minimum intensity requirement for $\mathrm{B}$ (ignoring spread of neural activation on the frequency dimension) is that it have the same spectrum level as $\mathrm{A}$ at all of A's frequencies. (The term spectrum level refers to the intensity of a designated part of the spectrum, without reference to the energy in any other part.) One purpose of the experiment was to establish that continuity could be obtained when the spectrum level of the WBN exactly matched that of the NBNs at all the frequencies of the latter. Since spectrum level is uniform across the band in the case of rectangular spectra, this purpose favored the use of such spectra.

The NBN stimuli, then, varied in CF, BW, and duration. We use the term bandwidth (i.e., BW) to refer to the upper band-edge frequency minus the lower band-edge frequency.

In order to create flat bands with very sharp band edges, all noises were created by the addition of sinusoids equal in amplitude but with random phases, closely spaced in frequency ( $1-\mathrm{Hz}$ increments). The NBNs were resynthesized (off line) with a new random seed for each condition of each listener, in order to average out idiosyncrasies in the particular noise samples. The WBNs were created in the same way and were flat from 300 to $5700 \mathrm{~Hz}$. The lowest and highest spectral components present in the NBNs were 500 and $5660 \mathrm{~Hz}$. Therefore, the WBN included the frequencies present in all NBNs. For each new listener and for each duration, 5 WBNs were synthesized afresh, and a random selection of one of these with the right duration was made on each trial.

All stimuli had 1-msec rises and falls of amplitude, gated by the first quarter of a sine wave for onsets and the second quarter for offsets. There was a slight overlap of adjacent noise bursts: The 1-msec decay of the earlier one did not start until the $1-\mathrm{msec}$ rise of the later one was complete. This seemed to eliminate perceptible clicks at the boundary.

The BW manipulation was established as follows. In preliminary studies, we discovered that a noise band of a fixed $B W$ became more pitch-like as the CF was raised. Since we were not sure whether the actual $\mathrm{BW}$ or this pitch-like quality (which we call pitch strength) would affect stream segregation most, we decided to ensure that a certain level of BW had the same pitch strength for every CF of the NBN bursts. We did this by adjusting the actual BWs as a function of the CFs of the NBNs. Preliminary tests showed that the BWs at different CFs had to be proportional to the critical bands at those CFs in order to have equal pitch strength. In effect, we created a variable that we could call pitch-strength-equated bandwidth. For convenience, we will refer to it simply as equated bandwidth (eqBW) category. All NBNs in the same eqBW category have approximately the same pitch strength, although their actual bandwidths will differ.

The design of noise bursts is shown in Table 1. Starting with an NBN centered at $1000 \mathrm{~Hz}$, we arbitrarily set its narrowest $\mathrm{BW}$ to $100 \mathrm{~Hz}$. Each higher level step of BW category was created by increasing

Table 1

Bandwidths Corrected to Equal Pitch Strength

\begin{tabular}{|c|c|c|c|c|c|c|c|}
\hline \multirow{2}{*}{$\begin{array}{c}\text { eqBW } \\
\text { Category }\end{array}$} & \multicolumn{6}{|c|}{ Center Frequency $(\mathbf{H z})$} & \multirow{2}{*}{$\begin{array}{l}\text { Strength } \\
\text { of Pitch }\end{array}$} \\
\hline & 1000 & 1190 & 1590 & 2120 & 2830 & 3780 & \\
\hline Very narrow & 100 & 110 & 140 & 193 & 274 & 376 & Very strong \\
\hline Narrow & 178 & 196 & 249 & 344 & 488 & 669 & Strong \\
\hline Medium & 316 & 348 & 442 & 610 & 866 & 1188 & Moderately strong \\
\hline Wide & 562 & 618 & 787 & 1085 & 1540 & 2113 & Less strong \\
\hline Very wide & 1000 & 1100 & 1400 & 1930 & 2740 & 3760 & Least strong \\
\hline
\end{tabular}

this BW repeatedly by a factor of 1.78 , yielding $5 \mathrm{BWs}$ centered at $\mathrm{CF}=1000 \mathrm{~Hz}$, with pitch strength decreasing as BW increased.

To obtain the BWs for the 5 higher CFs that were matched with the $1000-\mathrm{Hz}$ CFs on pitch strength, the BW for each of the $1000-\mathrm{Hz}$ NBNs was altered by expanding it in proportion to the critical band at the higher CF. For example, in Table 1, we can compare the BWs for the most saturated pitch condition (top row) for the 1000- and $3780-\mathrm{Hz}$ CFs. Since the critical band at $3780 \mathrm{~Hz}$ is about 3.76 times the size of the one at $1000 \mathrm{~Hz}$, its BW was set to $376 \mathrm{~Hz}$. Preliminary listening by the researchers and their colleagues indicated that this adjustment yielded approximately equally pitch-saturated bands of NBN. Table 1 shows 5 levels of pitch strength (as directly created by equal $\log$ steps of BW) and, for each one, BWs equated on pitch strength for the six bands that had different CFs.

As BW was widened, holding spectrum level constant, the noise, of course, had more energy and sounded louder. However, we made no attempt to equate the intensity of the NBN bursts that had different BWs. We decided, instead, to equate them on spectrum level, keeping them the same as the spectrum level of the wide-band interrupting bursts so that their illusory continuity through the latter would not be biased by different NBN/WBN ratios of spectrum levels (we believed that these ratios would be critical in determining illusory continuity). However, this decision left us with a problem: It has been claimed that intensity differences can serve as a basis for stream segregation. If correct, this would imply that we would not know whether any obtained stream segregation in Tasks 2 and 3 would be attributable to CF differences alone or in part to the intensity differences introduced by the compensation for pitch strength. However, it was not necessary to know this for the purposes of the present experiment. The goal of the study was to determine whether the same manipulations would favor integration and segregation in illusory continuity and in stream segregation. Since the stimuli were identical in Tasks 1 and 2 , this goal could be met whether or not we knew precisely which underlying properties of the $H$ and $L$ NBNs were responsible for the observed patterns of integration and segregation.

\section{Apparatus and Intensity}

The signals were synthesized using MITSYN 8.1 signal-processing software at 20,000 samples per second (Henke, 1990), output via a 16-bit D/A converter, and were low-pass filtered with a passive Tchebychev filter having a $3-\mathrm{dB}$ cutoff at $6500 \mathrm{~Hz}$ and a slope of $-142 \mathrm{~dB} /$ octave. An analog graphic equalizer was used to equalize the energy of the output of the headphones across the frequency range used in the experiment. The listeners were tested individually in an audiometric chamber. The sounds were presented binaurally over Sennheiser HD-480 headphones. The headphone output level for the WBN, calibrated using a flat-plate coupler, was $75 \mathrm{~dB}$ (B weighting). Because of varying BWs, the NBNs measured between 55 and $65 \mathrm{~dB}$ (B weighting).

\section{Design}

There were three tasks, each with separate listeners: (1) continuity judgment, (2) stream segregation with WBNs, and (3) stream segregation without WBNs. In each task, the two NBNs of a given trial always differed from one another in CF but were always the same in eqBW category and duration. The following factors varied across trials to yield 75 conditions for each task, presented in a newly randomized order to each of the 16 listeners doing that task: (1) $\mathrm{CF}$ separation of $H$ and $L$ NBNs (five levels: $3,8,13,18$, and 23 semitones), (2) eqBW category (five levels; i.e., which row of Table 1 was chosen), and (3) rate of presentation (three levels: 400,296 , and $220 \mathrm{msec} /$ cycle).

\section{Training}

The listeners were shown a schematic diagram of the stimuli for their task. The percepts they were to listen for were explained and il- 
lustrated by clear auditory examples. For the continuity task, they heard an alternation of high and low pure tones either connected by frequency glides (to illustrate continuity) or not so connected (to show discontinuity). The high and low tones were presented binaurally at too slow a rate to segregate. For the stream segregation task, the integrated single-stream percept was illustrated by a binaural alternation of high and low tones at a rate of presentation too slow to segregate, whereas stream segregation was illustrated at the same rate but with high and low tones presented to separate ears. The listeners were told these were not the same as the experimental stimuli but merely illustrated different types of perception, which were also described verbally and with diagrams.

After this, prior to the experimental trials, there were 18 practice trials (not scored), containing a new random selection, for each listener, of conditions. Then, there were two blocks of test trials, each consisting of one replication of each condition.

\section{RESULTS}

For each listener, the two replications of each condition were averaged yielding one score per condition. These scores ( 75 scores $\times 48$ listeners) were subjected to a three-way repeated measures analysis using the multivariate analysis of variance (MANOVA) model. Univariate results are reported (equivalent to a repeated measures ANOVA). Figure 2 shows the results for the three tasks. The $y$-axis represents ratings of continuity for the continuity task and represents ratings of stream segregation for the stream segregation task, but they are oriented so that a low score represents continuity of a single stream in both tasks. Each row of Figure 2 represents a different task, and each column represents a particular combination of variables. The plots are CF separation $\times$ eqBW category (Column 1), onset-to-onset time $\times$ eqBW category (Column 2 ), and CF separation $X$ onset-to-onset time (Column 3 ). For each column, the label for the $x$-axis of the three plots is given at the bottom of the column, and the legend for the symbols for a given column is given at the bottom of the column. The vertical height of each symbol is twice the pooled standard error derived from the MANOVA for the two-way interaction plotted in that graph.

\section{Continuity Judgment}

The results for illusory continuity are shown in the top row of Figure 2. The $y$-axis runs from 1 to 7 , where 1 means that the sounds connected smoothly (continuity) and 7 means they did not connect smoothly (discontinuity). All three main variables had significant effects. Continuity tended to be broken down by faster presentation (as shown in Cell B) $[F(2,30)=7.68, p=.002]$. It was also reduced by greater CF separations (as shown in Cells $A$ and $C$ ) $[F(4,60)=35.71, p<.00001]$. It was also broken down by wider eqBWs (as shown by the parameter in Cells A and $\mathrm{B})[F(4,60)=9.998, p<.0001]$. No interactions were significant $(p>.05)$.

\section{Streaming Judgment with WBNs Present}

On each trial, the listener gave a rating from 1 to 7 , where 1 meant it was easy to follow an up-and-down melody (integration) and 7 meant it was hard to follow it (seg- regation). The results are shown in the middle row of Figure 2 . The three main variables had significant effects that were similar to those in the continuity judgments. Integration of a single stream was broken down in favor of segregation by faster presentation (as shown in Cell E) $[F(2,30)=61.8, p<.00001]$. It was also disrupted by greater CF separation (shown in Cells D and F) $[F(4,60)=$ $36.49, p<.00001]$. Finally, integration was also reduced by wider eqBWs (as shown by the parameter in Cells D and $\mathrm{E})[F(4,60)=21.52, p<.00001]$.

In addition, there was one significant interaction, as shown in Cell F, between frequency separation and onsetto-onset time $[F(8,120)=2.61, p=.012]$. Beginning at the lowest frequency separation, as frequency separation increased, the segregation built up more strongly at high rates than at lower ones. However, at the higher frequency separations, the curve for the highest rate seemed to be reaching some limit. This interaction of frequency separation with rate of presentation is regularly found in experiments on stream segregation (Bregman, 1990, pp. 58-65). The failure to find a corresponding interaction in the continuity judgments points to a different role of speed in the two perceptual phenomena.

\section{Streaming Judgment Without WBNs}

The three main variables had significant effects that were similar to those in the other two tasks (see the bottom row of Figure 2). The continuity of a single stream was broken down in favor of segregation by faster presentation (as shown in Cell $\mathrm{H}$ ) $[F(2,30)=142.5, p<.00001]$. It was also disrupted by greater CF separation (shown in Cells $\mathrm{G}$ and I) $[F(4,60)=13.88, p<.00001]$. Finally, integration was also reduced by wider eqBWs (shown by the parameter in Cells $\mathrm{G}$ and $\mathrm{H})[F(4,60)=15.80, p<.0001]$.

There were three significant interactions. The first was the interaction between frequency separation and onsetto-onset time $[F(8,120)=5.08, p<.00001]$, shown in Cell I. This appears to have been due to the same reasons as in the stream segregation judgments with WBN present (Cell F): The effects of frequency separation were smallest at the slow rate, and the segregation at the high rate seemed to reach a maximum before the highest frequency separation was reached. The second interaction was between onset-to-onset time and eqBW category, as shown in Cell $\mathrm{H}$. It seems to have been due to the fact that the effect of eqBW category in breaking up the single stream happened most strongly at the intermediate rate $[F(8,120)=$ $3.25, p=.002]$. Finally, there was a weak three-way interaction between onset-to-onset time, CF separation, and eqBW category $[F(32,480)=1.51, p=.04]$. Given the large number of tests inherent in a three-way analysis, this result could have been due to chance. If real, it was possibly due to the particularly weak effects of frequency separation at the slowest rate and narrowest eqBW categories or to the particularly large effects of eqBW category at the medium rate and medium-frequency separations. Note that the effect was very small, accounting for about $2 \%$ of the systematic variance (i.e., of all the variance accounted 


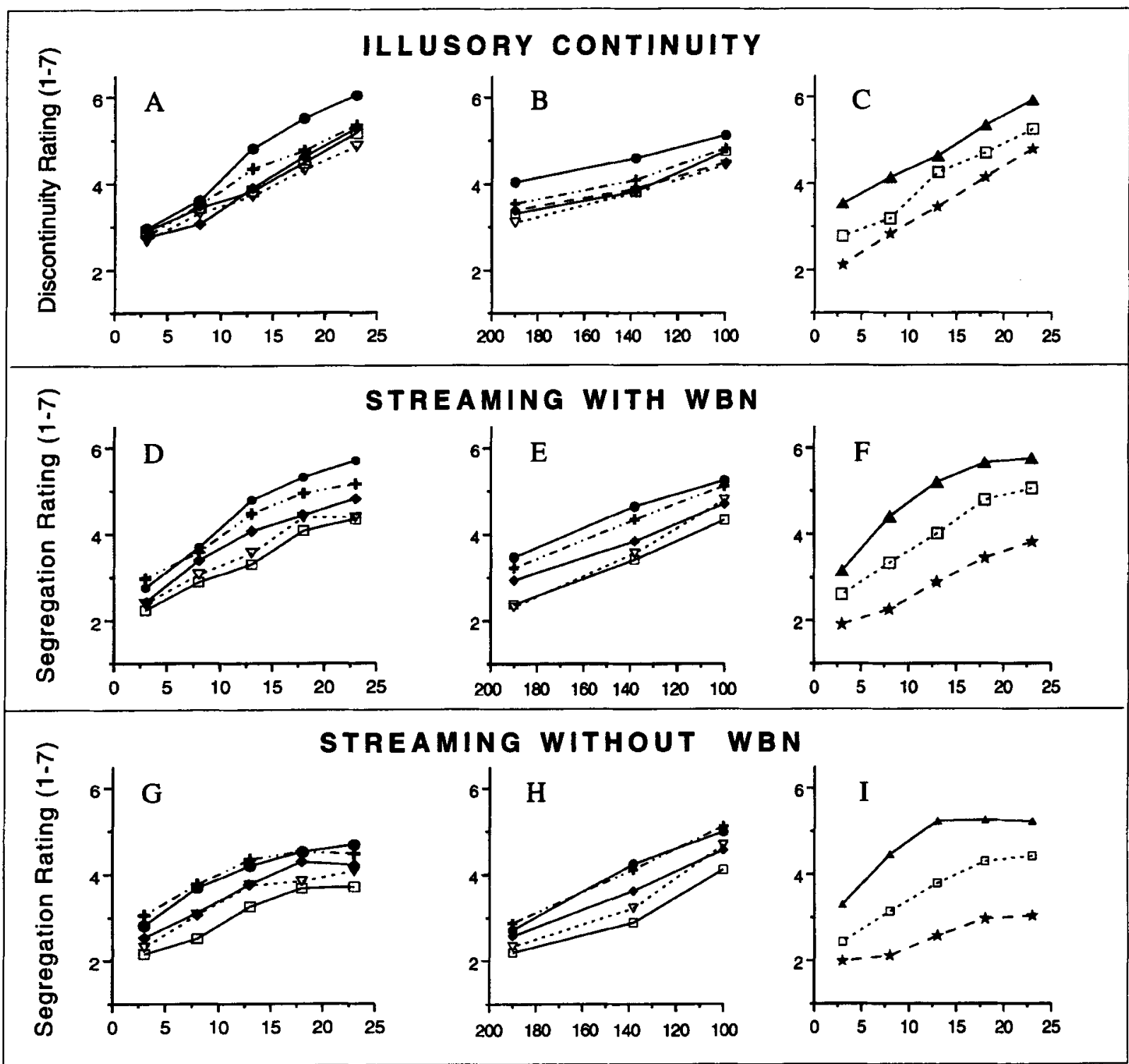

\section{Center Frequency Separation (semitones)}

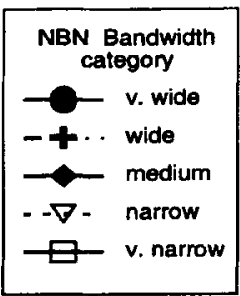

Onset-to-onset time (ms)

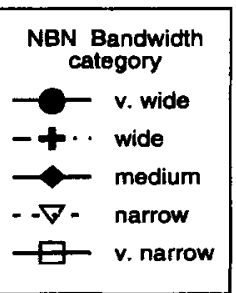

\section{Center Frequency Separation (semitones)}

\begin{tabular}{|c|c|}
\hline $\begin{array}{c}\text { Onset-to } \\
\text { time }\end{array}$ & $\begin{array}{l}\text {-onset } \\
\text { ms) }\end{array}$ \\
\hline 5 & 110 \\
\hline$-\square-$ & 148 \\
\hline$-\star$ & 200 \\
\hline
\end{tabular}

Figure 2. Each row shows results for a single task: illusory continuity, streaming with WBNs, or streaming without WBNs. Each column plots the rating of discontinuity for a particular combination of stimulus variables; high scores mean greater discontinuity. Left column: CF separation $\times$ eqBW category. Center column: onset-to-onset time $\times$ eqBW category. Right column: CF $\times$ onset-toonset time. The vertical height of each symbol is 2 times the pooled $S E$ for the interaction shown in that graph. 
for by the three variables and their interactions). Compare this with onset-to-onset time, which accounted for $55 \%$ of the systematic variance.

\section{Comparison of Stream Segregation Judgments With and Without WBN}

The two stream segregation tasks were compared in a separate analysis in which the stimulus type (presence of WBNs) was a between-subjects effect and onset-to-onset time, CF separation, and eqBW category were withinsubjects effects.

Since the effects of rate, $\mathrm{CF}$ separation, and eqBW category were significant when the two tasks were analyzed alone, they were even more strongly significant when the tasks were combined. For rate, the means, from fastest to slowest, were 4.76, 3.79, and $2.70[F(2,60)=178.2, p<$ $.00001]$. For CF separation, the means, from low to high CF, were $2.57,3.28,3.95,4.40$, and $4.55[F(4,120)=45.1$, $p<.00001]$. For eqBW category, the means, from very narrow to very wide, were $3.22,3.48,3.71,4.13$, and 4.22 $[F(4,120)=36.2, p<.00001]$.

There were some significant interactions. There was one between rate and eqBW category $[F(8,240)=3.74$, $p=.0006]$, with the greatest effects of eqBW category evident at the medium rate, as in the case of the stream segregation judgment without WBNs. Similarly, there was a significant interaction between rate and CF separation $[F(8,240)=7.18, p<.00001]$. However, the interaction between $C F$ separation and eqBW category was not significant $[F(16,480)=1.12, p=.34]$. None of the threeway interactions was significant at even the $20 \%$ level.

The most important comparisons were those involving the presence or absence of WBN. There was no main effect of the presence or absence of WBN (cf. Cells D, E, and $\mathrm{F}$ vs. Cells $\mathrm{G}, \mathrm{H}$, and I of Figure 2$)[F(1,30)=1.38$, $p=.25]$. The only significant effect involving WBN was a four-way interaction involving all variables $[F(32,960)=$ $1.74, p=.007]$, perhaps due to the weaker effects of CF separation in the no-WBN task, at the slowest rate, and particularly for the narrowest bands. However, this effect was very small, accounting for about $1 \%$ of all the systematic variance (the variance accounted for by the four variables and their interactions). Compare this with rate of presentation, which accounted for $47 \%$ of the systematic variance.

\section{Continuity Judgment Versus}

\section{Stream Segregation Judgment With WBN}

The two tasks carried out on the stimuli that contained WBNs - that is, the continuity and stream segregation judgments (top vs. middle row of Figure 2) -were compared in a single analysis, with judgment task as a between-subjects variable and with rate, CF separation, and eqBW category as within-subjects variables. The following variables significantly reduced integration: higher rates $[F(2,60)=39.0, p<.00001]$, larger $C F$ separations $[F(4,120)=71.6, p<.00001]$, and larger BWs $[F(4,120)=$
$28.2, p<.00001]$. There was also a significant CF separation $\times$ eqBW category interaction; the greater CF separations showed a stronger effect of BW category averaged over the two tasks $[F(16,480)=2.58, p=.001]$.

There was no significant main effect of task $[F(1,30)=$ $1.17, p>.29]$, but this fact shows only that the two groups of listeners centered their responses on the judgment scales in the same way. There were, however, two interactions that showed that some variables did affect the two tasks differently. First, there was a task $\times$ eqBW category interaction (cf. the clustering of the curves in Cells $\mathrm{A}$ and $\mathrm{D}$, or Cells B and E) $[F(4,120)=3.18, p=.02]$. It appears that $B W$ category (or the intensity difference correlated with it) had a slightly stronger effect on the stream segregation judgment. Figure 2 also suggests that the curves for different eqBW categories were more evenly spaced out for the stream segregation ratings than for the continuity ratings. Second, there was also a three-way interaction of task $\times$ rate $\times C F$ separation $[F(8,240)=$ $2.42, p=.02]$. In the continuity task (Cell C), the effect of $\mathrm{CF}$ separation was strongest at the slow rates, whereas in the stream segregation task (Cell F), this CF effect was strongest at the high rates, and the effects of $\mathrm{CF}$ separation on ratings seems to be approaching a maximum at this highest rate. Despite their statistical significance, these differences were quite small. We have no ready interpretation for them.

\section{DISCUSSION}

The first general observation is that stream segregation can occur with NBN stimuli and responds to the same variables as when it takes place with tonal stimuli. Segregation increases with separation of center frequencies between 3 and 23 semitones. This confirms results by Dannenbring and Bregman (1976), who used filtered noise with sharp spectral peaks rather than the (nominally) flat spectra of the present experiment. Our results extend those of Dannenbring and Bregman from the three levels of separation employed in their Experiment $1(1.65,8.14$, and 16.54 semitones) and the two levels (1.5 and 19 semitones) employed in their other experiments. We also found that segregation of NBNs increases with the rate of onsets for 110-, 150-, or 200-msec onset-to-onset time of NBNs (taking into account the silences or WBNs), confirming

\section{Table 2}

Bottom Frequency of Higher NBN (in Hertz) When the NBN With CF $=1000 \mathrm{~Hz}$ Is Alternated With a Higher CF NBN

\begin{tabular}{lcccccc}
\hline \multirow{2}{*}{$\begin{array}{c}\text { eqBW } \\
\text { Category }\end{array}$} & Lower CF of $1000 \mathrm{~Hz}$ & 1190 & 1590 & 2120 & 2830 & 3780 \\
\hline Very narrow & 1050 & 1135 & 1520 & 2023 & 2693 & 3592 \\
Narrow & 1089 & 1092 & 1466 & 1948 & 2586 & 3446 \\
Medium & 1158 & $1016^{*}$ & 1369 & 1815 & 2397 & 3186 \\
Wide & 1281 & $881^{*}$ & $1196^{*}$ & 1577 & 2060 & 2723 \\
Very wide & 1500 & $1140^{*}$ & $890^{*}$ & $1155^{*}$ & $1460 *$ & 1900 \\
\hline
\end{tabular}

*The upper CF band overlaps the lower 1000-Hz CF band. 
the results of Dannenbring and Bregman, who used 135and 185 -msec onset-to-onset times.

It should be mentioned that, in some cases, there was an overlap in frequency between the alternating high and low NBN bands. One might speculate that such an overlap should improve the continuity or hold the streams together better. These cases are marked by asterisks in Table 2. It is not evident that the ratings in these conditions are displaced toward values that are lower than those that one would expect from their positions in the overall pattern of results. Furthermore, the overall trends are visible even when these points are disregarded; so the presence of overlap in frequency did not appear to have distorted the results.

The same variables affected ratings of illusory continuity in a similar way. It can be seen, by comparing the plots in the same column of Figure 2, that each combination of variables had similar effects on the three tasks. While the magnitudes of the effects were not identical (e.g., eqBW category having a smaller effect on the continuity judgments) and the shapes of the curves were not identical (positive curvature in the stream segregation tasks, especially streaming without WBN), all effects were in the same direction and of similar magnitudes. The differences between the two streaming tasks were mainly that when WBN was present, segregation between high and low streams appeared to be stronger, the difference increasing with frequency separation. We can think of no convincing explanation for this effect.

The main difference between the ratings of stream segregation and those of illusory continuity is that the curves for stream segregation appear to be curvilinear when plotted against frequency separation. For ratings of stream segregation, increases of a fixed number of semitones in frequency separation had decreasing effects on rated stream segregation as frequency separation increased (Cells D, F, G, and I of Figure 2). Ratings of illusory continuity, on the other hand, showed approximately equal increases in mean ratings as frequency separation increased (Cells $\mathrm{A}$ and $\mathrm{C}$ of Figure 2). Also, whereas the segregation in both stream segregation tasks showed an interaction between frequency separation and rate of presentation, no such interaction was found in the illusory continuity task.

Apparently, there is a difference in the way the perception is taking place in the two tasks. However, this would be expected since different types of percepts are being formed. There is enough similarity to suggest that a common factor is involved. In the introduction, we suggested that (1) integration of all sounds into a single stream and (2) illusory continuity merely represent two ways of dealing with the links placed by bottom-up (stimulus-governed) grouping mechanisms between bits of acoustic evidence that should probably go together. We propose that it is the activity of this shared linking mechanism that is responsible for the similar effects of the three independent variables across the three tasks (but see our discussion of convergent operations, below).

Another observation concerns BW. Our original reason for employing different BWs was to extend the existing findings about the effects of frequency separation and rate of presentation to bands of noise with varying BWs. A glance at the first two columns of Figure 2 shows that wide and narrow bands respond in the same way to these variables. Neither stream segregation nor continuity requires very narrow-band stimuli.

We employed the two tasks-stream segregation with WBNs and stream segregation without WBNs-to find out whether adding WBN between the to-be-grouped sounds in a sequence of alternating $\mathrm{H}$ and L NBNs would have any effect on the grouping. Inspection of Figure 2 suggests a little more stream segregation when WBNs were present, and one might expect such an effect from the nature of the task: The listeners were asked to answer the question, "How difficult is it to hold onto the melody?" One might expect that, quite independently of stream segregation, the WBN bursts would make it more difficult to hold on to. However, the overall difference between the two sets of ratings was not statistically significant. In Figure 2, the ordinal properties of the curves for the two stream segregation tasks show similar effects of $\mathrm{CF}$ separation, eqBW category, and onset-to-onset time.

The fact that we obtained continuity when the NBNs were no softer than their corresponding frequencies in the WBN also has theoretical significance. It confirms the idea proposed in the introduction that the stimulus that provokes the perception of continuity requires only enough energy in the spectral region of an interrupting sound, $B$, to match the amount that is the present in the spectrum of the softer sound, A; this allows the matching neural activity to be removed from the sense data provided by $B$ and linked together sequentially with the sense data from $A$ to create the perception of an unbroken sound.

There are, however, some ambiguities in the results. Some of these have been subsequently resolved by a parallel study carried out to answer some of the questions raised by the present study (Bregman, Ahad, \& Van Loon, 1998). The first ambiguity comes from the fact that the different BWs for the alternated $\mathrm{H}$ and $\mathrm{L}$ noise bands, introduced to equate for pitch strength, led to differences in intensity between the two bands. We deliberately made no attempt to attenuate the wider BW NBNs to compensate for this difference because we wanted all bands to have the same spectrum level so that they would continue equally well through a fixed WBN that had a flat spectrum. That is, we wanted to equate the spectrum levels of each NBN with the WBN in the spectral region common to both, rather than equating the intensities of the NBNs themselves.

Van Noorden (1975) reported that an intensity difference between alternating tones promotes their segregation into separate streams, although it is the experience of the first author that the effects of intensity on stream segregation are very weak. Still, if one accepted van Noorden's observations as relevant for the NBNs of the present experiment, one might suspect that some of the effect of CF separation could have resulted from these intensity differences. However, in a study using NBNs that were not separated by WBNs, Bregman et al. (1998) found that CF separation 
has a very large effect on stream segregation-even larger than observed in the present experiment-when the BWs of the alternating noises are made equal on a log-frequency scale and equated for RMS intensity. Therefore, the observed CF effect of the present experiment was probably due directly to the $C F$ variable.

Another ambiguity concerns the observed effects of eqBW category. One might again suspect that the effect was due to intensity. This time it is not the intensity differences between $\mathrm{H}$ and $\mathrm{L}$ bands in the same cycle that is in question but intensity differences across levels of the eqBW variable. It should be pointed out that the eqBW variable was not correlated with differences in the intensity of $\mathrm{H}$ and $\mathrm{L}$ bands. For each $\mathrm{CF}$ separation, taken alone, the eqBW category manipulation can be thought of as a simple BW manipulation. As eqBW increased, there was an equal multiplication of the BWs for both the $\mathrm{H}$ and the $\mathrm{L}$ alternating NBNs, superimposed on the BW differences that were introduced in order to equalize pitch strength. Since any change of energy was proportional for $\mathrm{H}$ and $\mathrm{L}$ bands, their relative intensities should have remained unchanged. So eqBW category affects overall intensity, not intensity differences between the $\mathrm{H}$ and $\mathrm{L}$ bands. The authors are not aware of any research that shows a direct effect of overall intensity on stream segregation. In any case, the cited study by Bregman et al. (1998), in which all NBNs were equated on RMS energy, found no independent effect of BW category.

However, differences in overall intensity due to greater BW cannot explain the breakdown of illusory continuity with higher eqBW. It is fairly well established that it is the intensity of the matching frequency components in the louder interrupting sound and the softer interrupted sound that play a role in the induction of illusory continuity: Houtgast has argued that the sensory stimulation in any frequency region must not rise as the interrupting sound goes off and the softer sound replaces it (e.g., Houtgast, 1972). In the present experiment, when the wider band of NBN reached the WBN, the part of the WBN that matched the NBN in frequency always had the same energy as the NBN, whatever the BW of the NBN. This was the consequence of the flat spectra that were used and the matching of the spectrum levels of all noise bands. So the intensity relation between the NBN and the corresponding frequencies in the WBN was independent of the BW of the NBN. The change in intensity of the NBNs with increasing BW did not affect it. For this reason, the breakdown in illusory continuity with larger eqBWs was probably due to the same factor that caused the stream segregation to increase.

There is yet another logical possibility for explaining the effects of eqBW category: An increase in BW may somehow have increased the effective frequency separation between $\mathrm{H}$ and $\mathrm{L}$ bands, and this may have operated independently of our CF manipulation. For example, the important frequency separation between $\mathrm{H}$ and $\mathrm{L}$ bands might not be the difference between their CFs but the separation between the higher edge of the $\mathrm{H}$ band and the lower edge of the $\mathrm{L}$ band (i.e., their most distant edges).
While increasing the $B W$ reduces the separation of the closest edges at the same time as it increases the separation between the most distant edges, the effects may not be fully compensatory. The question of whether the position of the band edges would strongly influence stream segregation was addressed in the cited experiment by Bregman et al. (1998). It was found that the CF on logarithmic coordinates (as in the present experiment) was indeed the best predictor of segregation, better than the separation between the outer edges of the bands (i.e., the one between the higher edge of the $\mathrm{H}$ band and the lower edge of the $\mathrm{L}$ band) or between the inner band edges bands (the one between the lower edge of the $\mathrm{H}$ band and the higher edge of the $\mathrm{L}$ band).

\section{CONCLUSIONS}

The results, on the whole, showed that, with NBN stimuli, the same manipulations of temporal and frequency parameters had similar effects on three judgment tasks: illusory continuity, stream segregation with WBN separating NBN bursts, and stream segregation without interpolated WBN. We say similar, rather than identical, because there were some interactions between variables and some aspects of the shapes of the curves that differed. Nonetheless, the similarities were found despite the fact that different listeners were used, with each group told only about the phenomenon that they were to judge. These results add support to the idea that one of the prerequisites for illusory continuity (i.e., the connecting of two areas of spectral energy, $\mathrm{A} 1$ and $\mathrm{A} 2$, over time into a single perceived event) is that $\mathrm{A} 1$ and $\mathrm{A} 2$ must satisfy the requirements for being in the same stream (Bregman, 1990, p. 361). As outlined in the introduction, this is not the only requirement that must be met before illusory continuity can occur.

We are not making the claim that the percept of stream segregation is more fundamental than that of illusory continuity. Both of these are end products of perception, not underlying mechanisms. They probably represent different results of the description-building process (Bregman, 1977) that happens after the occurrence of the more lowlevel process of sequential integration that links sequential parts of the evolving spectrum. When the descriptionbuilding process places $\mathrm{A} 1$ and $\mathrm{A} 2$ into the same stream despite audible silences between them, we experience a stream that contains a number of discrete events, as in the typical stream segregation experiment. When there is no audible silence between successive bursts of a softer sound, and the interrupting sound contains energy that could connect $\mathrm{A} 1$ and $\mathrm{A} 2$, a description is built in which all the frequency information from $\mathrm{A} 1$ and $\mathrm{A} 2$ and the matching part of $B$ are grouped together and interpreted as a single long sound whose central portion is being masked by the louder sound; this yields the illusory experience of continuity. (Note that, in an illusory continuity experiment in which a soft sound rapidly alternates with a louder interrupting sound, the repetitions of the louder interrupting sound are assigned to a stream of their own, one that does 
have gaps in it, so that descriptions of a continuous stream and a discontinuous one are formed at the same time, using different parts of the same stimulus sequence.)

We believe that what is common to the phenomena of stream segregation and illusory continuity is the process of assigning links between parts of the evolving spectrum that probably came from the same sound-producing event(s) and that this is what accounts for similarities in the way they are affected by acoustic variables.

Finally, it must be acknowledged that the existence of one or two experiments, such as the present one and that of Tougas and Bregman (1990), cannot, in themselves, show that stream segregation and illusory continuity share an underlying mechanism. The method of converging operations must be employed more broadly. That is, a number of additional experiments must be done, each manipulating acoustic variables in a way that is known to affect stream segregation. These experiments might include cumulative effects of repetition on segregation of high and low sounds (Bregman, 1978b), the resetting of the cumulative process by a sudden change in spatial location (a resetting demonstrated by Anstis \& Saida, 1985, and by Rogers \& Bregman, 1998). Similarly, both phenomena should be influenced by the competition of spectral proximities (Bregman, 1978a) and by the ability of a preceding induction sequence, containing only the high sounds, to increase the tendency for high and low sounds to segregate (Rogers \& Bregman, 1993). All manipulations that increase stream segregation should disrupt illusory continuity. The present experiment supplies only one of the pieces of data required by the method of converging operations.

\section{REFERENCES}

ANstis, S., \& SAIDA, S. (1985). Adaptation to auditory streaming of frequency-modulated tones. Journal of Experimental Psychology: Human Perception \& Performance, 11, 257-271.

BozZI, P., \& Vicario, G. (1960). Due fattori di unificazione fra note musicali: La vicinanza temporale e la vicinanza tonale [Two factors in the integration of musical notes: Temporal proximity and tonal proximity]. Rivista di Psicologia, 54, 235-258.

Bregman, A. S. (1977). Perception and behavior as compositions of ideals. Cognitive Psychology, 9, 250-292.
Bregman, A. S. (1978a). Auditory streaming: Competition among alternative organizations. Perception \& Psychophysics, 23, 391-398.

Bregman, A. S. (1978b). Auditory streaming is cumulative. Journal of Experimental Psychology: Human Perception \& Performance, 4, 380-387.

Bregman, A. S. (1990). Auditory scene analysis: The perceptual organization of sound. Cambridge, MA: MIT Press.

Bregman, A. S., Ahad, P. A., \& VAN LoOn, C. (1998). Stream segregation of narrow-band noise bursts. Unpublished manuscript, Department of Psychology, McGill University.

Bregman, A. S., \& Campbell, J. (1971). Primary auditory stream segregation and perception of order in rapid sequences of tones. Journal of Experimental Psychology, 89, 244-249.

Dannenbring, G. L., \& Bregman, A. S. (1976). Stream segregation and the illusion of overlap. Journal of Experimental Psychology: Human Perception \& Performance, 2, 544-555.

HENKE, W. L. (1990). MITSYN: A synergistic family of high-level languages for time signal processing. Version 8 [Computer software]. Belmont, MA: Author.

Houtgast, T. (1972). Psychophysical evidence for lateral inhibition in hearing. Journal of the Acoustical Society of America, 51, 1885-1894.

Rogers, W. L., \& Bregman, A. S. (1993). An experimental evaluation of three theories of auditory stream segregation. Perception \& Psychophysics, 53, 179-189.

Rogers, W. L., \& Bregman, A. S. (1998). Cumulation of the tendency to segregate auditory streams: Resetting by changes in location and loudness. Perception \& Psychophysics, 60, 1216-1227.

THURLOW, W. R. (1957). An auditory figure-ground effect. American Journal of Psychology, 70, 653-654.

TougAS, Y., \& BREGMAN, A. S. (1990). Auditory streaming and the continuity illusion. Perception \& Psychophysics, 47, 121-126.

VAN NOORDEN, L. P. A. S. (1975). Temporal coherence in the perception of tone sequences. Doctoral dissertation, Eindhoven University of Technology.

van NoOrden, L. P. A. S. (1977). Minimum differences of level and frequency for perceptual fission of tone sequences ABAB. Journal of the Acoustical Society of America, 61, 1041-1045.

VICARIO, G. (1960). L'effetto tunnel acustico [The acoustic tunnel effect]. Rivista di Psicologia, 54, 41-52.

WARREN, R. M. (1982). Auditory perception: A new synthesis. New York: Pergamon.

WARREN, R. M. (1984). Perceptual restoration of obliterated sounds. Psychological Bulletin, 96, 371-383.

Warren, R. M., ObuSEK, C. J., \& ACKroff, J. M. (1972). Auditory induction: Perceptual synthesis of absent sounds. Science, 176, 1149-1151.

(Manuscript received July 3, 1996; revision accepted for publication February 16, 1998.) 